\title{
Linear Acoustic Waves in a Nonisothermal Atmosphere. II. Photospheric Resonator Model of Three-minute Umbral Oscillations
}

\author{
Jongchul Chae ${ }^{1}$ (i), Juhyung Kang ${ }^{1}$ (i), and Yuri E. Litvinenko ${ }^{2}$ (10) \\ ${ }^{1}$ Astronomy Program, Department of Physics and Astronomy, Seoul National University, Seoul 08826, Republic of Korea \\ ${ }^{2}$ Department of Mathematics, University of Waikato, P. B. 3105, Hamilton 3240, New Zealand \\ Received 2019 May 31; revised 2019 July 30; accepted 2019 August 18; published 2019 September 23
}

\begin{abstract}
The velocity oscillations observed in the chromosphere of sunspot umbrae resemble a resonance in that their power spectra are sharply peaked around a period of about three minutes. In order to describe the resonance that leads to the observed 3-minute oscillations, we propose the photospheric resonator model of acoustic waves in the solar atmosphere. The acoustic waves are driven by the motion of a piston at the lower boundary, and propagate in a nonisothermal atmosphere that consists of the lower layer (photosphere), where temperature rapidly decreases with height, and the upper layer (chromosphere), where temperature slowly increases with height. We have obtained the following results: (1) The lower layer (photosphere) acts as a leaky resonator of acoustic waves. The bottom end is established by the piston, and the top end by the reflection at the interface between the two layers. (2) The temperature minimum region partially reflects and partially transmits acoustic waves of frequencies around the acoustic cutoff frequency at the temperature minimum. (3) The resonance occurs in the photospheric layer at one frequency around this cutoff frequency. (4) The waves escaping the photospheric layer appear as upwardpropagating waves in the chromosphere. The power spectrum of the velocity oscillation observed in the chromosphere can be fairly well reproduced by this model. The photospheric resonator model was compared with the chromospheric resonator model and the propagating wave model.
\end{abstract}

Key words: magnetohydrodynamics (MHD) - waves - Sun: atmosphere - Sun: chromosphere - Sun: oscillations Sun: photosphere - sunspots

\section{Introduction}

Three-minute oscillations of intensity and velocity are commonly noticeable in the chromosphere of sunspot umbrae. Even though these three-minute umbral oscillations have mostly been observed through chromospheric lines such as the $\mathrm{Ca}$ II $\mathrm{H}$ and $\mathrm{K}$ lines (e.g., Beckers \& Tallant 1969), $\mathrm{H} \alpha$ (e.g., Giovanelli 1972), the Ca II IR triplet (e.g., Lites et al. 1982), Na I $\mathrm{D}_{1}$ and $\mathrm{D}_{2}$ (e.g., Kneer et al. 1982), the He I $\lambda 10830$ line (e.g., Lites 1986), and the Mg II $h$ and $k$ lines (Gurman 1987), the oscillations have been identified from photospheric lines as well (Lites \& Thomas 1985; Stangalini et al. 2012; Chae et al. 2017). There has been increasing evidence that the three-minute oscillations observed in the chromosphere represent the slow magnetoacoustic waves that propagate upward in the gravitationally stratified medium (e.g., Brynildsen et al. 2004; Centeno et al. 2006; Felipe et al. 2010; Tian et al. 2014; Cho et al. 2015). Khomenko \& Collados (2015) presented a comprehensive review of the relevant observations, analytical theories, and numerical simulations of the oscillations and waves in sunspots, providing an updated picture for understanding sunspot oscillations.

The most important property of the three-minute oscillations is the presence of peaks in the power spectrum of the velocity or intensity. The spatiotemporally averaged spectrum usually displays multiple peaks that are closely spaced in frequency, but when they are spatially and temporally well resolved, each power spectrum has a single peak (e.g., Brynildsen et al. 2003; Centeno et al. 2006; Chae et al. 2017, 2018). The physical origin of this peak is not yet resolved, even though a great number of theoretical models have been put forward. The proposed models may be categorized as the model of internally excited acoustic waves, the propagating wave model, and the resonator model. We briefly review these models below.
Acoustic waves in an atmosphere are subject to the acoustic cutoff arising from gravitational stratification (Lamb 1932). The concept of the acoustic cutoff is unambiguously established in an isothermal atmosphere, where the acoustic waves of frequencies higher than the acoustic cutoff frequency, which is often called Lamb frequency, can propagate, carrying wave energy, while the waves of lower frequencies cannot propagate. Acoustic waves may be internally excited by a localized disturbance inside the atmosphere (Lamb 1909; Kalkofen et al. 1994; Chae \& Goode 2015). Chae \& Goode (2015) showed that the waves of frequencies above the cutoff are generated with higher frequency waves moving out of the source region at faster group speeds, while lower frequency waves linger there for a while because of slow group speeds. After a while, the region oscillates with frequencies that are very close to the cutoff frequency, which is often called a wake. The power spectrum of the oscillation becomes sharply peaked around the cutoff if the spatial extent of the impulsive disturbance is large enough. This model of acoustic waves that are internally excited in an isothermal atmosphere, however, may not be applicable to the three-minute oscillations that are commonly observed in the solar atmosphere. Even though the impulsive disturbances that lead to 3-minute oscillations were reported by Kwak et al. (2016) and by Song et al. (2017), there is no observational evidence that such events occur inside the atmosphere frequently enough to explain the observed predominant oscillations.

Recent observations (Chae et al. 2017; Cho et al. 2019; Kang et al. 2019) rather support the notion that the sources of excitation are located below the atmosphere, as has commonly been modeled by the piston motion at the bottom of the atmosphere in previous theoretical studies (e.g., Fleck \& Schmitz 1991; Kalkofen et al. 1994; Sutmann \& Ulmschneider 1995). These 
studies found that regardless of the specific form of the piston driving, the oscillations with a frequency close to the cutoff prevail in high altitudes of the atmosphere. This oscillation frequency has often been regarded as the natural frequency of the atmosphere resulting from a resonant response or free atmospheric oscillations (Fleck \& Schmitz 1991; Kalkofen et al. 1994). Chae \& Goode (2015), however, made it clear that this "resonant" response is not like the resonance in a resonant cavity, but is simply due to the combined effect of the acoustic cutoff and the driving power spectrum. If the driving power spectrum is peaked at a frequency below the cutoff and decreases with frequency above the cutoff, only the waves with a frequency above the cutoff become propagating waves, and the waves with a lower frequency become evanescent waves that cannot carry wave energy. As a result, the oscillation spectrum at a high altitude is found to be peaked around the cutoff. This is the propagating wave model. This propagating wave model can provide another explanation of the peak frequency in the observed three-minute oscillations. Note that this model has originally been developed for an isothermal atmosphere, and was applied to real observations by Centeno et al. (2006). The real solar atmosphere, however, is not isothermal, which poses a problem in the propagating wave model.

The nonisothermal nature introduces further complexity in the acoustic waves in the solar atmosphere. Even though there have been studies of its effect on the propagation of waves (e.g., Sutmann \& Ulmschneider 1995), its full understanding requires a careful investigation of the properties of acoustic waves. In a nonisothermal atmosphere, the acoustic cutoff frequency is not even uniquely defined, and its physical interpretation is not so simple as in an isothermal atmosphere. Chae \& Litvinenko (2018) defined the acoustic cutoff frequency of a nonisothermal atmosphere as the lower limit of the frequencies where both pressure fluctuation and velocity fluctuation become oscillatory with height. The cutoff frequency of a temperature-decreasing layer is then found to be equal to the Lamb frequency at the bottom, and that of a temperature-decreasing layer is determined not only by the Lamb frequency at the bottom, but also by the temperature gradient. They also found that the acoustic cutoff frequency in a nonisothermal layer does not sharply separate the propagating wave solution and the nonpropagating wave solution, but marks the center of the frequency band where the transition from low acoustic transmission to high transmission takes place.

A real solar atmosphere is more complex than either the temperature-increasing layer or the temperature-decreasing layer, being characterized by the presence of a temperature minimum region. Typically, temperature decreases with height in the photosphere and reaches the minimum in the temperature minimum region, and increases with height in the chromosphere. This temperature structure together with density stratification may introduce wave reflection inside the atmosphere, and hence may cause resonance to occur, which has motivated the investigators to devise various models of resonant cavities or resonators in order to account for the resonant periods in the umbral oscillations. They include the Alfvén wave atmospheric resonator (Uchida \& Sakurai 1975), the fast-wave photospheric resonator (Scheuer \& Thomas 1981; Thomas \& Scheuer 1982), the slow-wave atmospheric resonator (Leibacher \& Stein 1981), the slow-wave chromospheric resonator (Zhugzhda \&
Locans 1981; Zhugzhda et al. 1983; Gurman \& Leibacher 1984; Settele et al. 2001), the fast-wave subphotospheric resonator (Zhugzhda 1984), and the slow-wave subphotospheric resonator (Zhugzhda 1984).

The chromospheric resonator model has received much more attention than the other resonator models. It predicts a series of peaks in the power spectrum that are closely spaced (about $1 \mathrm{mHz}$ interval) in frequency. According to Zhugzhda (2007), the lowest frequency originates from the temperature plateau, and the next lowest frequency from the cutoff frequency of the temperature minimum. The higher frequency peaks originates from the temperature structure of the atmosphere.

Staude et al. (1985) calculated the oscillations using the chromospheric resonator model, and showed that the observed spectrum of resonant peaks in the velocity and intensity of transition region lines observed by the Solar Maximum Mission $(S M M)$ spacecraft can be well explained by a gradient model of the umbral chromosphere. From the SMM observations of the Mg II $\mathrm{k}$ and $\mathrm{h}$ lines, Gurman (1987) detected the intensity oscillations that are characterized by three distinct peaks in the power spectra averaged over sunspot umbrae. He showed that the frequencies of these peaks agree well with the first three peaks predicted by the chromospheric resonator model. Christopoulou et al. (2003) detected three oscillating modes in the umbral oscillations that were simultaneously observed in the $\mathrm{H} \alpha$ and the Fe I $\lambda 5576$ line and suggested that the lowest frequency peaks are due to the photospheric resonator, while the two higher frequency peaks are due to the chromospheric resonator.

Botha et al. (2011) presented the results of numerical simulation of slow magnetoacoustic waves in an umbral model atmosphere that was excited by a velocity pulse beneath the photosphere. The chromosphere acts as a leaky resonator and the power spectrum of the velocity in the chromosphere has multiple peaks - at the frequency that corresponds to the acoustic cutoff of the temperature minimum at the higher frequencies. This finding supports the chromospheric resonator model.

Brynildsen et al. (2003, 2004), however, found that the power spectra of extreme ultraviolet (EUV) intensity above each sunspot umbra are characterized by one dominant peak that corresponds to a period of three minutes, and concluded that there is no indication of resonance frequencies that would be equally spaced $(1 \mathrm{mHz})$, as predicted by the chromospheric resonator model. Meanwhile, Zhugzhda \& Sych (2014) analyzed the $6 \mathrm{hr}$ run of He II $\lambda 304$ intensity over a sunspot umbra and obtained a very complex power spectrum with a dozen peaks. The problem posed by this observation is that the peaks are too numerous to be explained by the chromospheric resonator model. This model gives rise to only a few peaks, not a dozen. Thus it was concluded by Zhugzhda \& Sych (2014) and Zhugzhda (2018) that in addition to the chromospheric resonator, a subphotospheric resonator is needed to explain the observation.

Khomenko \& Collados (2015) noted that granting that a power spectrum has multiple peaks as seen in spatiotemporally averaged data, explaining these peaks using a resonator model is not easy. Primarily, the precise positions of the peaks change significantly from one observation to another, and even in time within the same observational sequence. This led Khomenko \& Collados (2015) to conclude that there is no 

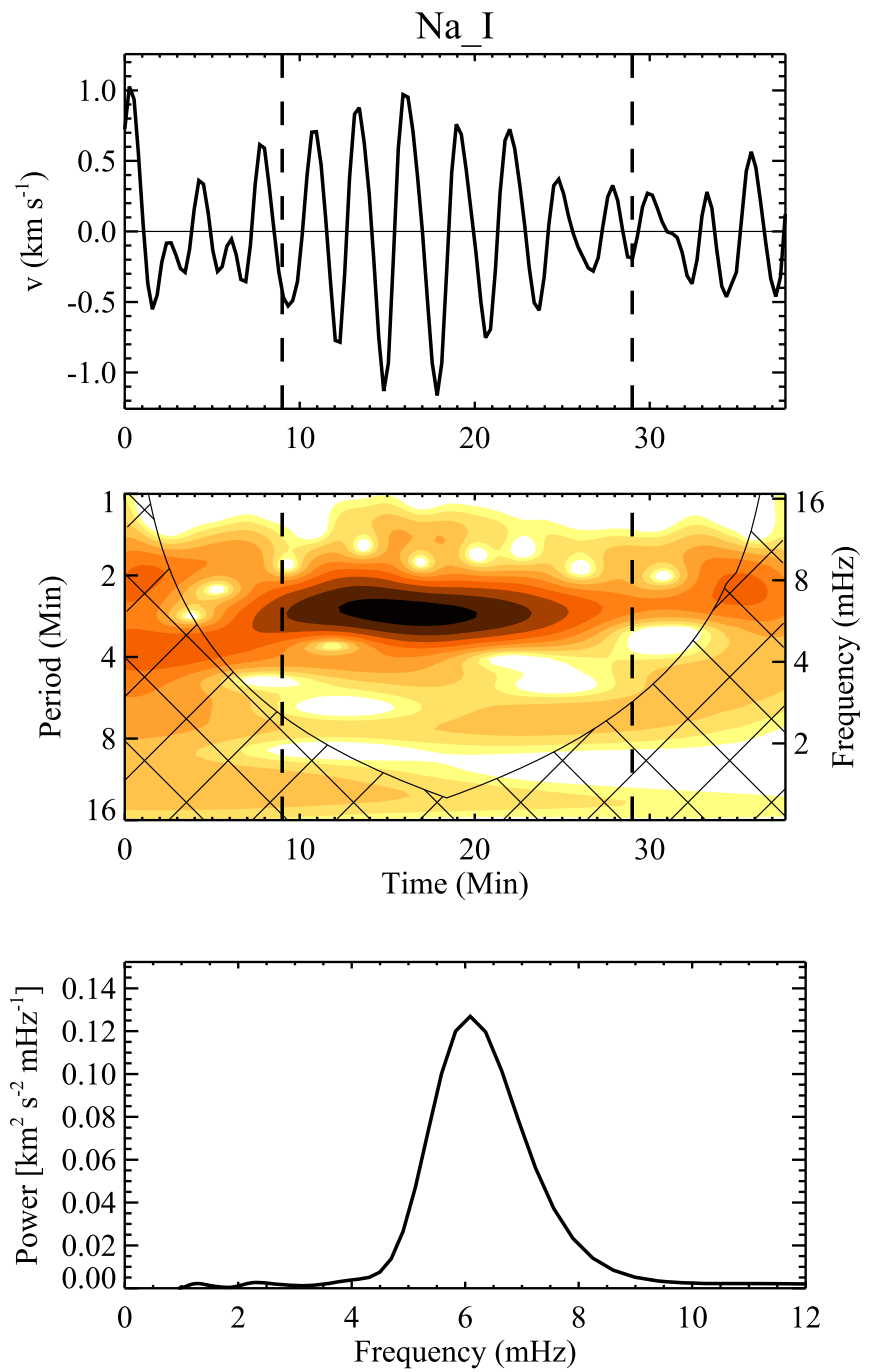

Figure 1. Plots of velocity oscillations measured inside a sunspot from the $\mathrm{Na}$ $\mathrm{D}_{2}$ line, and its wavelet power spectrum and global wavelet power spectrum. The two vertical dashed lines indicate the start and end of the wave duration. The temporal average of the wavelet power spectrum over the wave duration yields the global wavelet power spectrum.

strong observational support for an umbral resonance cavity model of any kind. By employing numerical simulations of wave propagation, Felipe (2019) investigated the propagating wave model and the chromospheric resonator model together, and concluded that the propagating wave model can explain the dominant period of the three-minute oscillations even without the chromospheric resonator, but the chromospheric resonator strongly enhances the power of the three-minute oscillations if it exists.

In this paper, we propose an alternative model as the physical origin of the peak frequency in the umbral oscillations. It is the photospheric resonator model of acoustic waves. This model is similar to the chromospheric resonator model and the subphotospheric resonator model in that the temperature minimum region plays an important role as a reflecting interface. The temperature minimum region reflects the waves of lower frequencies and transmits the waves of higher frequencies. Because wave driving is very likely to occur below the temperature minimum, a frequency-depending resonator cannot but be formed between the temperature minimum and the driving position. We set the wave driving

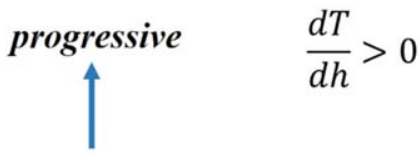

\section{T-minimum}

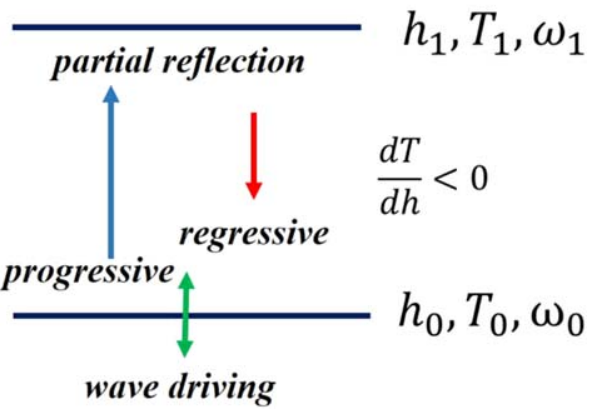

Figure 2. Illustration of the photospheric resonator. The waves of frequencies around $\omega_{1}$ are partially reflected by the interface at the temperature minimum.

near the bottom of the atmosphere. Thus the resonator basically spans the photosphere, having a thickness of a few hundred kilometers, being much smaller than the thickness of the subphotospheric resonator (Zhugzhda 1984), which is a few thousand kilometers.

The photospheric resonator is a leaky resonator in that the resonant oscillations escape it. In the chromosphere, the photospheric resonator model is very like the model of simple propagating waves because waves propagate upward there in both the models. An idea similar to our photospheric resonator was previously proposed by Taroyan \& Erdélyi (2008), but a detailed investigation of its physical concept and the comparison with specific observations was not reported.

We show that the photospheric resonator can successfully reproduce the oscillation power spectrum that is characterized by a single peak. We also compare the photospheric resonator model with the chromospheric resonator model and the propagating wave model.

\section{The Physical Picture of the Photospheric Resonator}

Figure 1 presents an example of velocity oscillations that are observed in the $\mathrm{NaI} \mathrm{D}_{2}$ line, which forms in the low chromosphere above a point inside a sunspot. The detailed description and the first analysis of the data were presented by Chae et al. (2017). The figure indicates that the three-minute velocity oscillation in the low chromosphere seen through the $\mathrm{Na}$ I line is characterized by the presence of a single dominant peak in the power spectrum. In this case, the frequency is around $6.1 \mathrm{mHz}$, which corresponds to a peak period of 2.7 minutes.

Figure 2 presents the graphical illustration of the model of a photospheric resonator. In this model, the atmosphere consists of two nonisothermal layers: the photospheric layer, and the chromospheric layer. Temperature rapidly decreases with height in the photosphere, and slowly increases with height in the chromosphere. The interface between the two layers is the temperature minimum.

In our previous work (Chae \& Litvinenko 2018, hereafter Paper I), we have defined the acoustic cutoff frequency in a nonisothermal atmosphere as the frequency separating the spatially oscillatory waves and the nonoscillatory waves. The acoustic waves of the cutoff frequency are partially transmitted and partially reflected. In a temperature-decreasing layer like 
the photospheric layer, the acoustic cutoff frequency is equal to the Lamb frequency specified by the sound speed $c_{0}$ at its bottom,

$$
\omega_{0}=\frac{\gamma g}{2 c_{0}}
$$

where $\gamma$ is the specific heat ratio, and $g$ is the gravitational acceleration. In contrast, in a temperature-increasing layer like the chromospheric layer, the acoustic cutoff frequency is affected by the temperature gradient as well; it is slightly higher than the Lamb frequency specified by the sound speed $c_{1}$ at its bottom,

$$
\omega_{1} \equiv \frac{\gamma g}{2 c_{1}}
$$

Note that the acoustic cutoff frequency of the chromosphere is close to $\omega_{1}$ because the chromospheric layer in our model is close to an isothermal layer.

Our model implies $\omega_{0}<\omega_{1}$. Therefore it is expected that the waves of $\omega \ll \omega_{0}$ exist only in the interior because the bottom of the photospheric layer does not transmit them into the photospheric layer. The interface, i.e., the temperature minimum region, plays the role of the reflecting top of the photospheric layer. It fully reflects back the low-frequency waves of $\omega \ll \omega_{1}$. The waves of $\omega \sim \omega_{1}$ are partially reflected back, which causes the interference of the progressive waves and the regressive waves, and the resonance with the waves driving at frequencies near $\omega_{1}$. As a result, both the progressive waves and regressive waves are amplified, and the progressive waves leaking out of the interface contain large power at frequencies near $\omega_{1}$. Note that because the reflection is partial, the power of the progressive waves should be larger than that of the regressive waves, and a net upward flux of wave energy exists even inside the photospheric resonator. In contrast, the interface little reflects the waves of $\omega \gg \omega_{1}$ so that resonance does not take place. Consequently, the power of the progressive waves in the chromosphere may be small at $\omega \gg \omega_{1}$ unless the power of wave driving in the photosphere is strong at these frequencies.

An important assumption of the photospheric resonator we propose is that there exists a lower boundary where the velocity is no longer determined by the internal dynamics of the resonator, but is externally controlled, being prescribed by a piston motion. The location of the wave driving can be parameterized by its temperature $T_{0}$ because the temperature monotonically increases with depth in the photosphere and in the interior.

\section{Multilayer Modeling of Acoustic Waves in a Nonisothermal Atmosphere}

\subsection{Equilibrium Model}

We consider an atmosphere that is in hydrostatic equilibrium,

$$
\frac{d \ln p}{d z}=-\frac{\gamma g}{c^{2}}
$$

and consists of several layers. The gravitational acceleration $g$ is assumed to be constant. As is well known, the sound speed $c$ is related to pressure $p$, density $\rho$, and temperature $T$,

$$
c=\sqrt{\frac{\gamma p}{\rho}}=\sqrt{\gamma R T},
$$

with $R \equiv k_{B} / \mu m_{\mathrm{H}}$, where $k_{B}$ is the Boltzmann constant, $\mu$ is the mean molecular weight, and $m_{\mathrm{H}}$ is the hydrogen mass.

One can freely choose the reference pressure $p_{r}$ that specifies the reference atmospheric level, and the corresponding sound speed $c_{r}$ at the reference level. By introducing the scale length

$$
H_{r} \equiv \frac{2 c_{r}^{2}}{\gamma g},
$$

which is equal to twice the local pressure scale height at the reference level, we define the reduced height,

$$
h \equiv-\frac{1}{2} H_{r} \ln \frac{p}{p_{r}},
$$

which is measured from the reference level. Note that we measure the geometrical height $z$ from this reference level as well. In the special case of an isothermal atmosphere, $h$ is equal to $z$.

In Paper I we have considered the wave solution in a simple layer where $d c^{2} / d h$ is constant. We generalize this condition to the multilayer atmosphere. We require that it is constant in each layer indexed by $l$ and is specified by the dimensionless parameter $\kappa_{l}$, which is defined as

$$
\kappa_{l} \equiv \frac{H_{r}}{c_{l}^{2}} \frac{d c^{2}}{d h}
$$

for $h_{l} \leqslant h<h_{l+1}$, where $h_{l}$ refers to the bottom of the $l$ th layer, and $c_{l}$ is the sound speed there. This equation is integrated to give

$$
\frac{c^{2}}{c_{l}^{2}}=1+\kappa_{l} \frac{h-h_{l}}{H_{r}} .
$$

By combining Equations (3), (5), (6), and (8), we obtain the relationship between $h$ and $z$ :

$$
\frac{d z}{d h}=\left(1+\kappa_{l} \frac{h-h_{l}}{H_{r}}\right) \frac{c_{l}^{2}}{c_{r}^{2}},
$$

which is integrated to give

$$
z-z_{l}=\left(h-h_{l}\right)\left(1+\frac{1}{2} \kappa_{l} \frac{h-h_{l}}{H_{r}}\right) \frac{c_{l}^{2}}{c_{r}^{2}},
$$

and, after inversion

$$
h-h_{l}=\frac{H_{r}}{\kappa_{l}}\left(-1+\sqrt{1+2 \frac{\kappa_{l}}{H_{r}} \frac{c_{r}^{2}}{c_{l}^{2}}\left(z-z_{l}\right)}\right) .
$$

\subsection{Wave Solution in Each Layer}

The general solution for the linear acoustic waves can be easily constructed using the principle of superposition. We express pressure fluctuation and velocity fluctuation for each layer indexed by $l$ as

$$
\Delta p(h, t ; l)=p^{\frac{1}{2}}(h) \int_{-\infty}^{\infty} \psi_{\omega}(h ; l) \exp (-i \omega t) d \omega
$$




$$
v(h, t ; l)=p^{-\frac{1}{2}}(h) \int_{-\infty}^{\infty} \varphi_{\omega}(h ; l) \exp (-i \omega t) d \omega
$$

using the complex wave functions $\psi_{\omega}(h ; l)$ and $\varphi_{\omega}(h ; l)$ that are written as

$$
\begin{aligned}
& \psi_{\omega}(h ; l)=C_{l}\left(f_{\omega}(h ; l)+r_{\omega, f_{\omega}} f_{\omega}(h ; l)\right) \\
& \varphi_{\omega}(h ; l)=D_{l}\left(g_{\omega}(h ; l)+r_{\omega, l} g_{\omega}^{*}(h ; l)\right),
\end{aligned}
$$

where $f_{\omega}(h ; l)$ and $g_{\omega}(h ; l)$ in general represent the progressive components of pressure fluctuation and velocity fluctuation, respectively, and their conjugates $f_{\omega}^{*}(h ; l)$ and $g_{\omega}^{*}(h ; l)$, the regressive components. The reflection coefficient $r_{\omega, l}$ is the coefficient ratio of the regressive component to the progressive component.

Note that Equations (12) and (13) are similar to Equations (18) and (19) of Paper I, but are different from these in that Equations (12) and (13) are the full Fourier transform representation, while Equations (18) and (19) of Paper I are a simple summation representation. This difference mostly accounts for the difference in the formulae and expressions between the current work and our previous one in Paper I.

According to Paper I, in the $l$ th layer, the coefficients $C_{l}$ and $D_{l}$ are related to each other as

$$
\frac{D_{l}}{C_{l}}=\frac{i g}{2 \omega} .
$$

Following Paper I, we change the independent variable $h$ in the $l$ th layer into a new dimensionless variable $\zeta$ that is related to $\omega$, $h$ and $l$,

$$
\zeta \equiv \frac{1}{a_{l}^{2}}\left(\frac{\omega^{2}}{\omega_{l}^{2}}-1\right)+\frac{a_{l}}{H_{r}}\left(h-h_{l}\right),
$$

with the Lamb frequency at the bottom of the lth layer

$$
\omega_{l}=\frac{\gamma g}{2 c_{l}}
$$

and a dimensionless parameter $a_{l}$ defined as

$$
a_{l}=s_{l}\left(\left|\kappa_{l}\right| \frac{\omega^{2}}{\omega_{l}^{2}}\right)^{1 / 3},
$$

where $s_{l}$ is the sign of $\kappa_{l}$. Note that $a_{l}$ depends on $\omega$ as well as $\kappa_{l}$ and $\omega_{l}$. It is obvious that the value of $\zeta$ at the bottom of the and after some arrangements, one can show that $\zeta$ at the top of the same layer is given by

$$
\zeta\left(\omega, h_{l+1} ; l\right)=\frac{1}{a_{l}^{2}}\left(\frac{\omega^{2}}{\omega_{l+1}^{2}}-1\right) .
$$

Note that $h$ is continuous across the interface between the $l$ th layer and $l+1$ th layer, but $\zeta$ is not in general: $\zeta\left(\omega, h_{l+1} ; l\right) \neq \zeta\left(\omega, h_{l+1} ; l+1\right)$ unless $a_{l}=a_{l+1}$.

It has been shown in Paper I that the equation describing the function $f(\zeta)=f_{\omega}(h ; l)$ is given by

$$
\frac{d^{2} f}{d \zeta^{2}}+\zeta f=0
$$

and the progressive solution representing the waves propagating upwards is given by

$$
f(\zeta)=f_{J}(\zeta)+i s_{l} f_{Y}(\zeta),
$$

with two real function $f_{J}$ and $f_{Y}$ that can be expressed in terms of Bessel functions and modified functions. These two functions can also be expressed in terms of the Airy functions:

$$
\begin{gathered}
f_{J}(\zeta)=\frac{3}{2} \operatorname{Ai}(-\zeta)-\frac{\sqrt{3}}{2} \operatorname{Bi}(-\zeta) \\
f_{Y}(\zeta)=-\frac{\sqrt{3}}{2} \mathrm{Ai}(-\zeta)-\frac{3}{2} \operatorname{Bi}(-\zeta) .
\end{gathered}
$$

Note that $f_{J}$ is a real function that behaves like a sine function at $\zeta>0$, and $f_{Y}$ is a real function that behaves like a negative cosine function (see Figure 4 of Paper I). Once $f(\zeta)$ is determined, $g_{\omega}(h ; l)=g(\zeta)$ can be determined from $f_{\omega}$ using the equation

$$
g(\zeta)=f(\zeta)-a_{l} \frac{d f(\zeta)}{d \zeta}
$$

The behavior of the functions $f(\zeta)$ and $g(\zeta)$ at three different regimes can be conveniently investigated using the asymptotic expressions

$$
f(\zeta) \approx \begin{cases}\sqrt{\frac{3}{\pi}} \zeta^{-\frac{1}{4}} e^{i s_{l} \theta} & \text { for } \zeta>1 \\ 0.7765 \zeta+s_{l}(0.4483 \zeta-1.2299) & \text { for }-1<\zeta<1 \\ \sqrt{\frac{3}{\pi}}|\zeta|^{-\frac{1}{4}} \exp \left(\frac{2}{3}|\zeta|^{\frac{3}{2}}\right) e^{-(2 \pi / 3) s_{l} i} & \text { for } \zeta<-1\end{cases}
$$

and

$l$ th layer $\left(h=h_{l}\right)$ is given by

with

$$
\zeta\left(\omega, h_{l} ; l\right)=\frac{1}{a_{l}^{2}}\left(\frac{\omega^{2}}{\omega_{l}^{2}}-1\right),
$$

$$
\begin{aligned}
& \text { for } \zeta>1 \\
& \text { for }-1<\zeta<1 \\
& \text { for } \zeta<-1
\end{aligned}
$$




\subsection{Interface and Boundary Conditions}

We consider the atmosphere composed of $L$ layers. At the interface $h=h_{l+1}$ between the $l$ th layer and the $l+1$ th layer, both $\psi_{\omega}$ and $\varphi_{\omega}$ should be continuous:

$$
\begin{aligned}
& C_{l}\left(f_{\omega}\left(h_{l+1} ; l\right)+r_{\omega} f_{\omega}^{*}\left(h_{l+1} ; l\right)\right) \\
& \quad=C_{l+1}\left(f_{\omega}\left(h_{l+1} ; l+1\right)+r_{\omega, l+1} f_{\omega}^{*}\left(h_{l+1} ; l+1\right)\right) \\
& D_{l}\left(g_{\omega}\left(h_{l+1} ; l\right)+r_{\omega, l} g_{\omega}^{*}\left(h_{l+1} ; l\right)\right) \\
& \quad=D_{l+1}\left(g_{\omega}\left(h_{l+1} ; l+1\right)+r_{\omega, l+1} g_{\omega}^{*}\left(h_{l+1} ; l+1\right)\right)
\end{aligned}
$$

for $l=0, . ., L-2$. We also impose the top boundary condition that only progressive waves exist in the top layer indexed by $l=L-1$ :

$$
r_{\omega, L-1}=0,
$$

and the bottom boundary condition that the velocity at the bottom is given by

$$
\varphi_{\omega}\left(h_{0} ; 0\right)=D_{0}\left(g_{\omega}\left(h_{0} ; 0\right)+r_{\omega, 0} g_{\omega}^{*}\left(h_{0} ; 0\right)\right),
$$

where $\varphi_{\omega}\left(h_{0} ; 0\right)$ is related to $v\left(h_{0}, t ; 0\right)$ by the equation

$$
\varphi_{\omega}\left(h_{0} ; 0\right)=\frac{1}{2 \pi} p_{0}^{\frac{1}{2}} \int_{-\infty}^{\infty} v\left(h_{0}, t ; 0\right) \exp (i \omega t) d t,
$$

which has been derived from Equation (13).

In order to model a pulse of finite duration, we consider the propagation of the wave packet produced by the vertical displacement of the form

$$
\xi\left(h_{0}, t\right)=-\xi_{0} \exp \left[-\frac{1}{2}\left(\frac{t-t_{0}}{t_{p}}\right)^{2}\right],
$$

corresponding to the velocity of a piston

$$
v\left(h_{0}, t\right)=\frac{\xi_{0}}{t_{p}} \frac{t-t_{0}}{t_{p}} \exp \left[-\frac{1}{2}\left(\frac{t-t_{0}}{t_{p}}\right)^{2}\right]
$$

at the low boundary. According to this velocity model, the piston is at rest at $t=-\infty$, is accelerated downward, and reaches the maximum displacement $\xi_{0}$ at $t=t_{0}$ with zero velocity. After this, the piston is accelerated upward, and reaches the original equilibrium position at $t=\infty$. The disturbance is characterized by the maximum displacement $\xi_{0}$ and the dynamic timescale of the pulse $t_{p}$.

By inserting this model into Equation (34), we obtain the expression of $\varphi_{\omega}\left(h_{0} ; 0\right)$

$$
\varphi_{\omega}\left(h_{0} ; 0\right)=i \exp \left(i \omega t_{0}\right) \frac{\xi_{0} p_{0}^{1 / 2}}{\sqrt{2 \pi}} t_{p} \omega \exp \left(-\frac{1}{2} \omega^{2} t_{p}^{2}\right) .
$$

\section{Theoretical Results from a Two-layer Model}

\subsection{A Two-layer Model Approximation}

Figure 3 presents the plot of $c^{2} / c_{r}^{2}$ versus $h / H_{r}$ constructed from the values of pressure and density in the mean umbral model of Maltby et al. (1986). Here we set the reference level of $h=z=0$ at the surface where the optical depth at $500 \mathrm{~nm}$ is unity. The normalizing parameters $c_{r}$ and $H_{r}$ were calculated with pressure and density at this reference level.

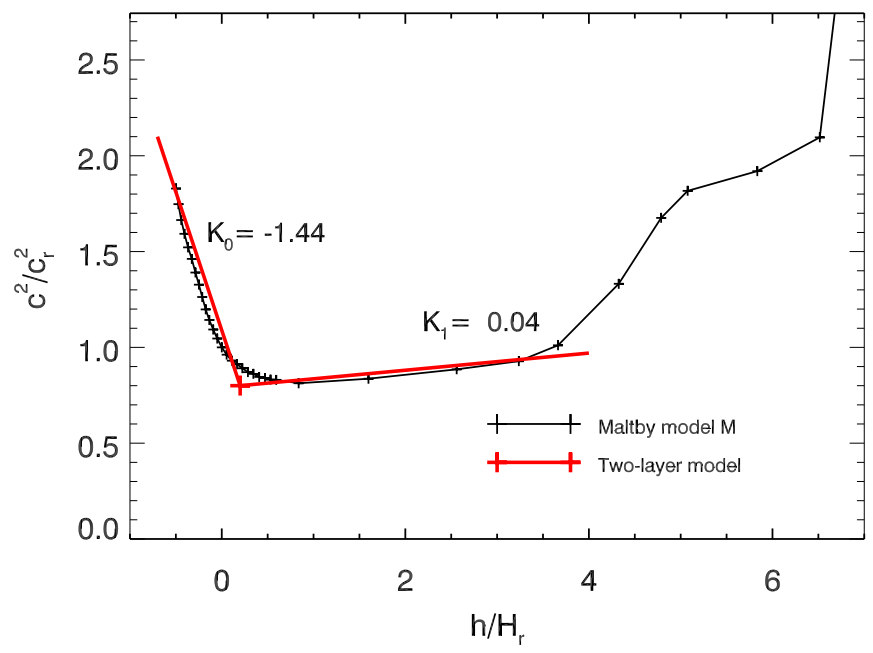

Figure 3. Plots of sound speed square $c^{2}$ (i.e., proportional to temperature) vs. reduced height $h$ constructed from the umbral model of Maltby et al. (1986).

The figure clearly indicates that $c^{2}$ rapidly decreases with $h$ in the photosphere, and then very slowly increases with $h$ in the chromosphere. We find that this variation can be fairly well approximated by a two-layer model $K_{0}=-1.44$ and $K_{1}=0.04$ with the interface of $c_{1}^{2} / c_{r}^{2}=0.8$ being at $h_{1} / H_{r}=0.2$, where $K_{l}$ is the dimensionless gradient in the lth layer, defined by

$$
K_{l} \equiv \frac{H_{r}}{c_{r}^{2}} \frac{d c^{2}}{d h} .
$$

From Equations (7) and (38), we obtain the relationship

$$
\kappa_{l}=\frac{c_{r}^{2}}{c_{l}^{2}} K_{l},
$$

which indicates $\kappa_{1}=K_{1} / 0.8=0.05$.

The bottom of the photospheric layer is regarded as a free parameter of the model. To specify it, we introduce the dimensionless parameter

$$
\tau \equiv \frac{c_{0}^{2}}{c_{1}^{2}},
$$

which is equal to the ratio of temperature $T_{0} / T_{1}$ when $\gamma R$ is constant. Once $\tau$ is known, we can calculate $c_{0}$, and hence $\kappa_{0}$ using Equation (39). Then by inserting $l=0, c=c_{1}$, and $h=h_{1}$ into Equation (8) and by solving it for $h_{0}$, we obtain

$$
h_{0}=h_{1}-\left(\frac{c_{1}^{2}}{c_{0}^{2}}-1\right) \frac{H_{r}}{\kappa_{0}} .
$$

In addition, by inserting $l=0, h=z=0$ into Equation (10) and by solving it for $z_{0}$, we obtain the expression

$$
z_{0}=h_{0}\left(1-\frac{1}{2} \kappa_{0} \frac{h_{0}}{H_{r}}\right) \frac{c_{0}^{2}}{c_{r}^{2}},
$$

and by inserting $l=0, h=h_{1}, z=z_{1}$ into Equation (10) and by solving it for $z_{1}$, we obtain the expression

$$
z_{1}=z_{0}+\left(h_{1}-h_{0}\right)\left(1+\frac{1}{2} \kappa_{0} \frac{h_{1}-h_{0}}{H_{r}}\right) \frac{c_{0}^{2}}{c_{r}^{2}} .
$$




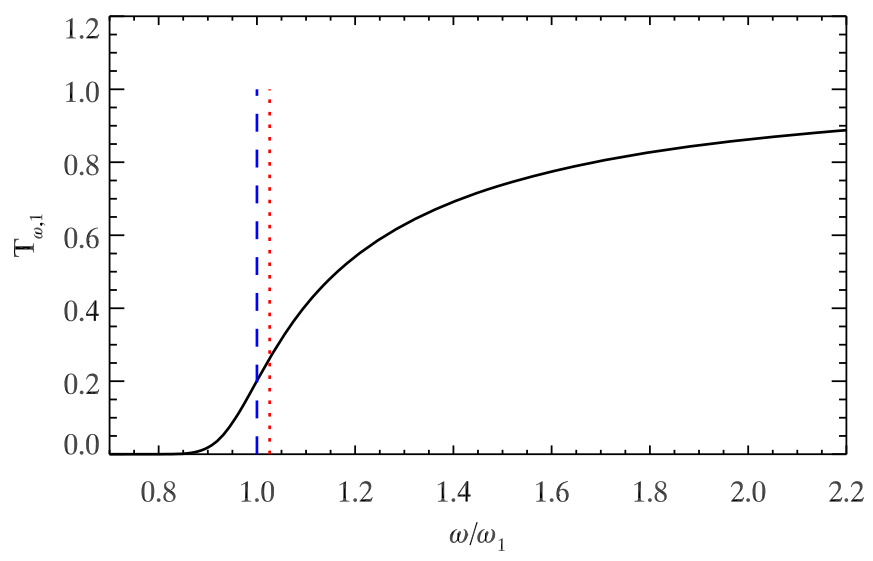

Figure 4. Plot of the transmission efficiency $T_{\omega}$ of the upper layer. The vertical blue dashed line refers to the Lamb frequency $\omega_{1}$, which corresponds to $c_{1}$, and the vertical red dotted line refers to the acoustic cutoff frequency $\omega_{\mathrm{ac}}$. Note that $T_{\omega}$ is above zero even at frequencies below $\omega_{1}$ and $\omega_{\mathrm{ac}}$, which is a characteristic of the nonisothermal layer.

\subsection{Acoustic Cutoff and Transmission Efficiency of the Chromospheric Layer}

In our two-layer model, the chromospheric layer is a simple nonisothermal layer where waves propagate upward. There are no regressive waves in this layer. In Paper I we have considered the acoustic transmission efficiency of a simple nonisothermal layer where only progressive waves exist. The transmission efficiency $T_{\omega}$ of such a layer was defined by the ratio of the wave flux to the maximum value that can be realized when pressure and velocity fluctuations are perfectly correlated. Because the upper chromospheric layer indexed by $l=1$ is a temperature-increasing layer where only progressive waves exist, its acoustic cutoff frequency is given by

$$
\omega_{\mathrm{ac}}=\frac{\omega_{1}}{\sqrt{1-\kappa_{1}}}
$$

which is equal to $1.03 \omega_{1}$. According to Paper $\mathrm{I}$, the transmission efficiency of this layer is given by

$$
T_{\omega}=\frac{3}{\pi} \frac{\left|a_{1}\right|}{\left|f_{\omega}\left(h_{1} ; 1\right)\right| \cdot\left|g_{\omega}\left(h_{1} ; 1\right)\right|} .
$$

Figure 4 presents the plot of $T_{\omega}$ versus $\omega$. We find from the figure that there is a smooth transition of $T_{\omega}$ from zero to unity at frequencies around $\omega_{\mathrm{ac}}$ and the waves transport energy even at frequencies lower than $\omega_{\mathrm{ac}}$. This is the property of the acoustic cutoff in a nonisothermal layer, which was reported in Paper I.

Note that $T_{\omega}$ is the property of the layer. It is wholly determined by the parameters $\omega_{1}$ and $\kappa_{1}$ of the upper layer, and is not at all affected by the parameters of the lower layer. In contrast, the reflection coefficient to be discussed in the following is the property of the interface, characterized by the difference in the parameters between the lower layer and the upper layer.

\subsection{Reflection at the Interface}

In the case of the two-layer model with $L=2$, one can derive from Equations (30)-(32) the expression of the

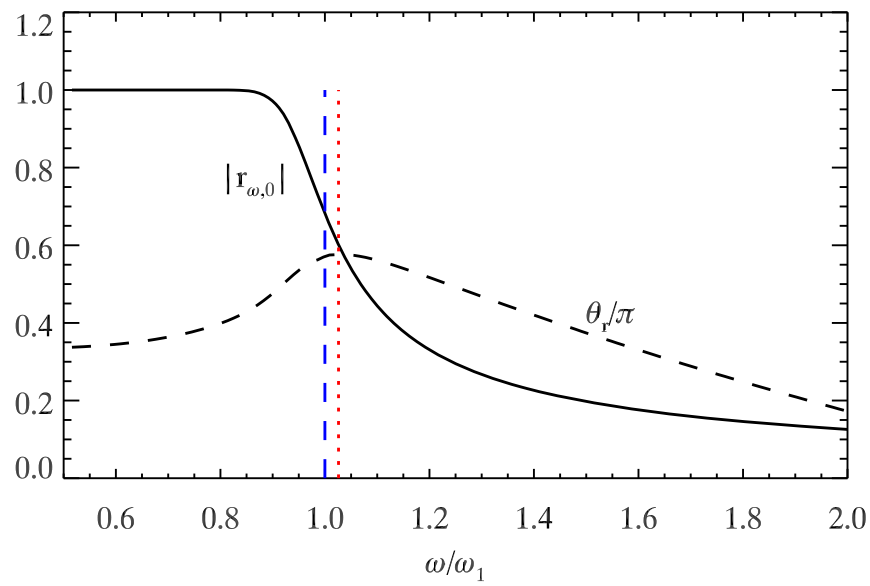

Figure 5. Plots of the absolute value (solid black curve) and phase (dashed curve) of the reflection coefficient $r_{\omega, 0}$ as functions of $\omega$. The vertical blue dashed line refers to the Lamb frequency $\omega_{1}$, which corresponds to $c_{1}$, and the vertical red dotted line refers to the acoustic cutoff frequency $\omega_{\mathrm{ac}}$.

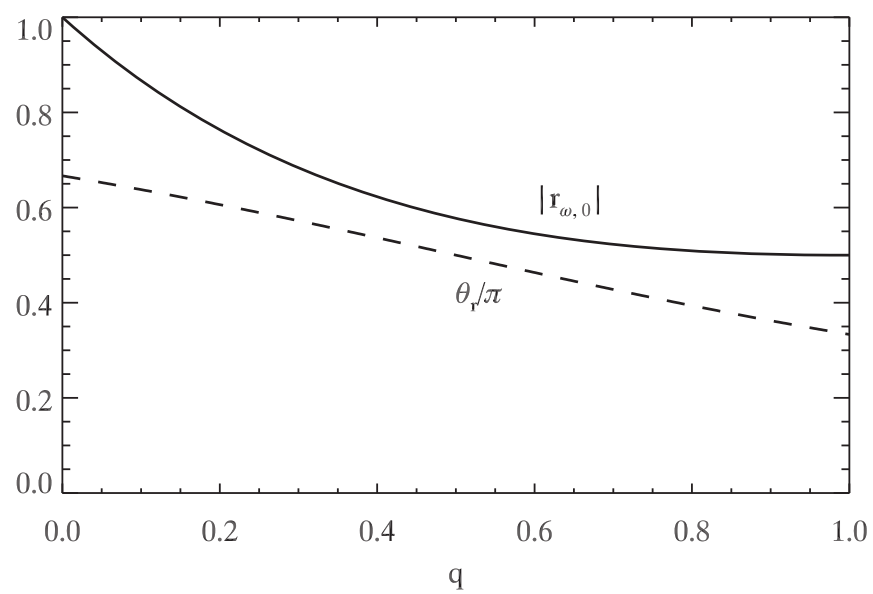

Figure 6. Plots of the amplitude $\left|r_{\omega, 0}\right|$ and phase $\theta_{r}$ of the reflection coefficient as functions of the atmospheric model parameter $q$ at the frequency $\omega=\omega_{1}$.

reflection coefficient $r_{\omega, 0}$ in the lower layer:

$$
r_{\omega, 0}=\frac{f_{\omega}\left(h_{1} ; 1\right) g_{\omega}\left(h_{1} ; 0\right)-f_{\omega}\left(h_{1} ; 0\right) g_{\omega}\left(h_{1} ; 1\right)}{f_{\omega}^{*}\left(h_{1} ; 0\right) g_{\omega}\left(h_{1} ; 1\right)-f_{\omega}\left(h_{1} ; 1\right) g_{\omega}^{*}\left(h_{1} ; 0\right)},
$$

with the magnitude and phase defined by

$$
r_{\omega, 0}=\left|r_{\omega, 0}\right| e^{i \theta_{r}} .
$$

Figure 5 presents the plots of $r_{\omega, 0}$ computed using Equation (46) as a function of $\omega$. The plots indicate that $\left|r_{\omega, 0}\right|$ rapidly decreases with $\omega$ at frequencies around $\omega_{1}$. We find from the figure that $\left|r_{\omega, 0}\right|$ is anticorrelated with $T_{\omega}$. Note that $r_{\omega, 0}$ depends on the parameters of the lower layer but $T_{\omega}$ does not. Thus, the strong anticorrelation between $\left|r_{\omega, 0}\right|$ and $T_{\omega}$ may be understood only if they are very sensitive to the parameters that commonly affect both. The comparison of Figures 4 and 5 suggests that both the parameters are very much affected by the acoustic cutoff frequency of the chromospheric layer $\omega_{\mathrm{ac}}$, which is practically determined by the minimum temperature of the atmosphere.

To understand how $r_{\omega, 0}$ depends on the parameters of the two layers, we examine Equation (46) in the special case of $\omega=\omega_{1}$. This choice leads to the following expressions: $\zeta\left(\omega_{1}\right.$, $\left.h_{1} ; 0\right)=\zeta\left(\omega_{1}, h_{1} ; 1\right)=0, a_{0}=-\left(\left|\kappa_{0}\right| \tau\right)^{1 / 3}$, and $a_{1}=\left|\kappa_{1}\right|^{1 / 3}$. 
It then follows that $f_{\omega 1}\left(h_{1} ; 0\right)=1.2299 i, f_{\omega 1}\left(h_{1} ; 1\right)=$ $-1.2299 i, \quad g_{\omega_{1}}\left(h_{1} ; 0\right)=1.2299 i+(-0.7765+0.4483 i) a_{0}$, and $g_{\omega_{1}}\left(h_{1} ; 1\right)=-1.2299 i+(-0.7765-0.4483 i) a_{1}$. After some algebra, we obtain the expression

$$
r_{\omega_{1}, 0}=\frac{-(1-q) /(1+q)+0.5773 i}{1+0.5773 i},
$$

with the parameter

$$
q \equiv-\frac{a_{1}}{a_{0}}=\left(\frac{\left|\kappa_{1}\right|}{\left|\kappa_{0}\right| \tau}\right)^{1 / 3}=\left(\frac{\left|K_{1}\right|}{\left|K_{0}\right|}\right)^{1 / 3} .
$$

Figure 6 presents the plots of the magnitude $\left|r_{\omega, 0}\right|$ and phase $\theta_{r}$ of c calculated with $\omega=\omega_{1}$ is calculated as functions of $q$ using Equation (48). The figure clearly indicates that both $\left|r_{\omega, 0}\right|$ and $\theta_{r}$ decrease with $q$. The lower the value of $q$, the stronger the reflection. This means that for a fixed $K_{1}$, a high value of $\left|K_{0}\right|$ causes strong reflection at the interface. Namely, the more rapidly the temperature decreases with height in the photosphere, the more strongly are the waves reflected at the temperature minimum.

An important point is that $q$ only weakly depends on $K_{1} / K_{0}$. In the specific model we consider, $K_{0}=-1.44$ and $K_{1}=0.04$, so that we have $q=0.30$. Even if $K_{1} / K_{0}$ were to increase or decrease by a factor of 2 , the range of $q$ would be confined to the range from 0.24 to 0.38 , in which case $\left|r_{\omega_{1}, 0}\right|$ would be still in the range from 0.63 to 0.73 , little deviating from the value of 0.68 calculated with the specific model. Therefore we conclude that the reflection coefficient is only weakly affected by the specific structure of the atmospheric model. It is primarily determined by $\omega_{1}$.

It is interesting that the reflection coefficient $r_{\omega_{1}, 0}$ is completely determined by one parameter $q$ or by the ratio $K_{1} / K_{0}$. From this result we expect that $r_{\omega, 0}$ at other frequencies is also controlled by $K_{1} / K_{0}$. This can be explicitly demonstrated for $\omega$ close to $\omega_{1}$ when the asymptotic formula for $f(\zeta)$ yields a correction to the reflection coefficient $r_{\omega, 0}$, which is on the order of $\left(\omega^{2} / \omega_{1}^{2}-1\right) q$. Thus the dependence of $r_{\omega, 0}$ on $a_{0}^{2}$ and $a_{1}^{2}$ is indeed relatively weak. It is somewhat simpler to analyze the dependence of $r_{\omega, 0}$ on $\omega$ if it is expressed in terms of the function $f(\zeta)$ and its derivatives:

$$
r_{\omega, 0}=-\frac{f_{\omega}\left(h_{1} ; 1\right) f_{\omega}^{\prime}\left(h_{1} ; 0\right)+q f_{\omega}\left(h_{1} ; 0\right) f_{\omega}^{\prime}\left(h_{1} ; 1\right)}{f_{\omega}\left(h_{1} ; 1\right) f_{\omega}^{* \prime}\left(h_{1} ; 0\right)+q f_{\omega}^{*}\left(h_{1} ; 0\right) f_{\omega}^{\prime}\left(h_{1} ; 1\right)} .
$$

This form makes it obvious, for instance, that $\left|r_{\omega, 0}\right|=1$ when $q=0$, as expected physically.

\subsection{Resonance in the Photospheric Layer}

The reflection at the temperature minimum produces the regressive waves, and the interference between the regressive waves and the progressive waves at the bottom of the photospheric layer results in a resonance at a specific frequency. Thus the photospheric layer plays the role of a resonator. We assume that the bottom of the photospheric resonator is subject to a piston driving, and its velocity can be set to a prescribed complex function $\varphi_{\omega}\left(h_{0} ; 0\right)$ as in Equation (37). Then from Equation (33), one can derive the amplitude coefficient $D_{0}$ :

$$
D_{0}=D_{0,0} \frac{1}{1+\left|r_{\omega, 0}\right| e^{i\left(\theta_{r}-2 \theta_{0}\right)}},
$$

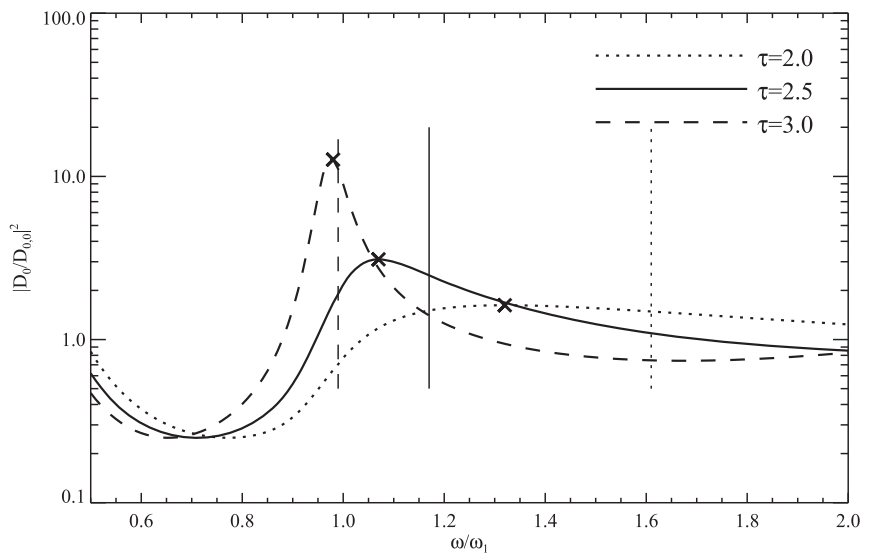

Figure 7. Plots of normalized amplitude coefficient square $\left|D_{0} / D_{0,0}\right|^{2}$ in the photospheric layer as functions of frequency $\omega$ in the different cases of the model parameter $\tau$. The symbols mark the resonance frequency $\omega_{R}$ and the peak values. The vertical lines mark the value of $\omega_{M} / \omega_{1}$ satisfying Equation (55).

where $D_{0,0}$ is the value of $D_{0}$ in the limiting case of no reflection given by

$$
D_{0,0} \equiv \frac{\varphi_{\omega}\left(h_{0} ; 0\right)}{g_{\omega}\left(h_{0} ; 0\right)}
$$

and $\theta_{0}$ is the phase angle of $g_{\omega}\left(h_{0} ; 0\right)$ defined by

$$
g_{\omega}\left(h_{0} ; 0\right)=\left|g_{\omega}\left(h_{0} ; 0\right)\right| e^{i \theta_{0}} \text {. }
$$

Therefore

$$
\left|\frac{D_{0}}{D_{0,0}}\right|^{2}=\frac{1}{1+\left|r_{\omega, 0}\right|^{2}+2\left|r_{\omega, 0}\right| \cos \left(\theta_{r}-2 \theta_{0}\right)},
$$

which indicates that if $\left|r_{\omega, 0}\right|$ is not zero and is constant over $\omega$, the amplitude $\left|D_{0}, / D_{0,0}\right|^{2}$ has a local peak at the frequency $\omega_{M}$ satisfying the condition

$$
\theta_{r}-2 \theta_{0}=\pi,
$$

with which it follows $\cos \left(\theta_{r}-2 \theta_{0}\right)=-1$. This peak represents the resonance of the waves resulting from the interference between the progressive waves and regressive waves. Note that because $\left|r_{\omega, 0}\right|$ in fact varies with $\omega$, the resonance frequency of Equation (54), denoted by $\omega_{R}$, will actually differ from $\omega_{M}$.

Figure 7 presents the plots of $\left|D_{0}, / D_{0,0}\right|^{2}$ as a function of $\omega$ and indicates the values of $\omega_{R}$ and $\omega_{M}$ in the three different cases of $\tau$. Each resonance frequency $\omega_{R}$ lies lower than the corresponding $\omega_{M}$ because $\left|r_{\omega, 0}\right|$ decreases with $\omega$. The figure clearly shows that the higher value of $\tau$ corresponds to a lower value of $\omega_{R}$ and a sharper peak of $\left|D_{0}, / D_{0,0}\right|^{2}$. The values of $\omega_{R} / \omega_{1}$ are $1.32,1.07$, and 0.98 for $\tau=2.0,2.5$, and 3.0, respectively. It also should be noted that the resonance frequency $\omega_{R}$ has a value close to $\omega_{1}$ in all cases.

\subsection{Energy Spectra and Flux Spectra}

The time-averaged energy of the waves $\mathcal{E}(h ; l)$ at a height $h$ is given by

$$
E(h ; l)=\rho(h ; l) \frac{1}{t_{\mathrm{wv}}} \int_{-\infty}^{\infty} v^{2}(h, t ; l) d t,
$$

where $t_{\mathrm{wv}}$ is the duration of the wave packet or the length of the time interval where the velocity fluctuation has an amplitude 
significantly above zero. In the same way, the time-averaged flux of the wave energy is given by

$$
\mathcal{F} \equiv \frac{1}{t_{\mathrm{wv}}} \int_{-\infty}^{\infty} v \Delta p d t
$$

By applying Parseval's theorem and making use of the fact that the amplitude is an even function of $\omega$, we obtain

$$
E(h ; l)=\rho \frac{1}{t_{\mathrm{wv}}} \int_{0}^{\infty} \frac{4 \pi}{p}\left|\varphi_{\omega}(h ; l)\right|^{2} d \omega \equiv \int_{0}^{\infty} E_{\omega}(h ; l) d \omega
$$

and

$$
\mathcal{F}=\frac{1}{t_{\mathrm{wv}}} \int_{0}^{\infty} 4 \pi \Re\left[\varphi_{\omega} \psi_{\omega}^{*}\right] d \omega \equiv \int_{0}^{\infty} F_{\omega}(h ; l) d \omega .
$$

These expressions lead us to define the energy spectrum, velocity power spectrum, and flux spectrum as follows:

$$
\begin{gathered}
E_{\omega}(h ; l)=\frac{4 \pi \rho}{p t_{\mathrm{wv}}}\left|\varphi_{\omega}(h ; l)\right|^{2} \\
P_{\omega}(h ; l)=\frac{E_{\omega}(h ; l)}{\rho} \\
F_{\omega}=\frac{4 \pi}{t_{\mathrm{wv}}} \mathfrak{R}\left[\varphi_{\omega} \psi_{\omega}^{*}\right] .
\end{gathered}
$$

By inserting Equations (14) and (15), and making use of Equation (16), we obtain the expressions

$$
\begin{gathered}
t_{\mathrm{wv}} E_{\omega}(h ; l)=\frac{4 \pi \rho}{p}\left|D_{l}\right|^{2}\left|g_{\omega}+r_{\omega, l} g_{\omega}^{*}\right|^{2} \\
t_{\mathrm{wv}} F_{\omega}=\frac{24 \omega}{g}\left|D_{l}\right|^{2}\left(1-\left|r_{\omega, l}\right|^{2}\right)\left|a_{l}\right| .
\end{gathered}
$$

This formula for flux spectrum $F_{\omega}$ is derived making use of the relationship ${ }^{3}$

$$
f_{J} g_{Y}-f_{Y} g_{J}=-\frac{3}{\pi} a_{l}
$$

which results from the Wronskian formulae for the Airy functions.

Note that $E_{\omega}$ in Equation (63) depends on $h$ and $l$. Figure 8 shows the plots of $E_{\omega}$ calculated using Equation (63) at different heights in the specific case of $\tau=2.5$. We find from Figure 8 that a slight enhancement of $E_{\omega}(h) / E_{\omega}\left(h_{0}\right)$ over unity is detected around the resonance frequency $\omega_{R}$ near the bottom of the photospheric resonator. The strength of the energy enhancement increases with height, and reaches a peak at the top of the resonator $h / H_{r}=0.20$. The strength decreases with height at the upper heights $h / H_{r}>0.2$. Thus the photospheric resonator has a node at its bottom end $\left(h / H_{r}=-0.63\right)$, and an antinode at its top $\left(h / H_{r}=0.20\right)$. Figure 8 also shows that the energy spectra are affected by the acoustic cutoff. At low frequencies $\omega, E_{\omega}(h) / E_{\omega}\left(h_{0}\right)$ decreases with height and becomes much smaller than unity at heights $h / H_{r}>0.20$. As a consequence of this cutoff, the profile of $E_{\omega}(h) / E_{\omega}\left(h_{0}\right)$ at a large height, i.e., $h / H_{r}=2.52$, appears asymmetric over $\omega$, rapidly increasing with $\omega$ for $\omega \lesssim \omega_{1}$ and slowly decreasing with $\omega$ for $\omega>\omega_{1}$.

\footnotetext{
3 This relationship is the same as Equation (65) of Paper I, in which the minus sign was erroneously omitted, however.
}

\section{$\mathrm{E}_{\omega}(\mathrm{h}) / \mathrm{E}_{\omega}\left(\mathrm{h}_{0}\right)$ in case $\tau=2.50$}



Figure 8. Plots of normalized energy density spectra $E_{\omega}(h) / E_{\omega}\left(h_{0}\right)$ at different heights in the case of $\tau=2.5$ The energy density is enhanced near $\omega_{1}$. The enhancement increases with height and reaches maximum at the top of the photospheric resonator.

Figure 9 presents the plots of $F_{\omega}$ calculated using Equation (64). Note that $F_{\omega}$ is constant over $h$ in the $l$ th layer. It is in fact independent of $l$ as well, as expected from the principle of energy conservation. The reference flux spectrum $F_{\omega, 0}$ used in the plots is the flux spectrum of acoustic waves in a homogeneous medium:

$$
F_{\omega, 0} \equiv c_{0} E_{\omega}\left(h_{0} ; 0\right) .
$$

The plot of $F_{\omega} / F_{\omega, 0}$ in the case of $\tau=2.5$ is qualitatively similar to the plot of $E_{\omega}(h) / E_{\omega}\left(h_{0}\right)$ at the height $\left(h-h_{1}\right) / H_{r}=2.52$ (see Figure 8). We also find that the plots of $F_{\omega} / F_{\omega, 0}$ in Figure 9 look similar to those of $\left|D_{0} / D_{0,0}\right|^{2}$ in Figure 7 , respectively, except for the low frequencies. These similarities support the notion that the peak of $F_{\omega} / F_{\omega, 0}$ as well as that of $E_{\omega}(h) / E_{\omega}\left(h_{0}\right)$ results from the resonance in the photospheric layer and the effect of acoustic cutoff.

\section{Modeling the Observations}

\subsection{Atmospheric Model}

In this section, we fit the observed oscillations of the $\mathrm{NaI}$ line velocity presented in Figure 1. Note that the velocity data 




Figure 9. Plots of normalized wave energy flux spectra $F_{\omega} / F_{\omega, 0}$ in the different cases of $\tau$. The deeper the driving position, the sharper the energy flux spectrum.

have been taken from a specific point inside the umbra of a sunspot-the point marked by $\mathrm{P}$ in Chae et al. (2017). This point is located in a region where numerous umbral dots exist. It is well known that umbral dots are hotter than the diffuse umbral background (Solanki 2003). Because the umbral model of Maltby et al. (1986) describes the darkest regions in large sunspot umbrae, we cannot directly use the structure of $c^{2}$ in this model. Instead, to set the atmospheric model of the umbral dot region, we adopt the structure of $c^{2} / c_{r}^{2}$ in the umbral model as presented in Figure 3 and multiply it by the value of $c_{r}^{2}$ that is suitable for the umbral dot region. The sound speed of the surface in the model is $c_{r}=6.45 \mathrm{~km} \mathrm{~s}^{-1}$, which corresponds to the surface temperature of $4040 \mathrm{~K}$, but we adopt $c_{r}=$ $7.0 \mathrm{~km} \mathrm{~s}^{-1}$, which corresponds to $4740 \mathrm{~K}$ for the umbral dot region, which is consistent with the reported observation that umbral dots are on average $700 \mathrm{~K}$ hotter than the umbral minimum (Tritschler \& Schmidt 2002). In addition, we adopt the surface pressure $p_{r}=2.7 \times 10^{5} \mathrm{dyn}^{-2}$ as in the umbral model. The specification of the two-layer model of the umbral dot region is thus completed by the adoption of the relationship between $c^{2} / c_{r}^{2}$ and $h / H_{r}$, and the choice of $c_{r}$ and $p_{r}$. With this choice of $c_{r}$, we obtain $c_{1}=\sqrt{0.8} c_{r}=6.26 \mathrm{~km} \mathrm{~s}^{-1}$ and $\omega_{\mathrm{ac}}=$ $0.038 \mathrm{rad} \mathrm{s}^{-1}$. This angular frequency corresponds to the normal cutoff frequency of $5.97 \mathrm{mHz}$ or to the cutoff period of 2.79 minutes.

\subsection{The Na I Line Formation Height}

The formation height of the $\mathrm{Na}$ I line should be known for the observed velocity oscillations to be compared with the theory. Chae et al. (2017) estimated the formation height of the line at $680 \mathrm{~km}$ by analyzing the phase differences among the different spectral lines by making use of the progressive wave solution in the piecewise isothermal atmosphere. We now understand that the waves may not be fully progressive in the low atmosphere because of the reflection near the temperature minimum, and hence the above estimate may deviate from the real formation height. Nevertheless, we adopt $z_{\mathrm{D}}=680 \mathrm{~km}$ as an estimate of the formation height of the $\mathrm{Na}$ I line in the umbra because we have no other reference as yet. In this regard, this choice should be considered as a working hypothesis in the present work.

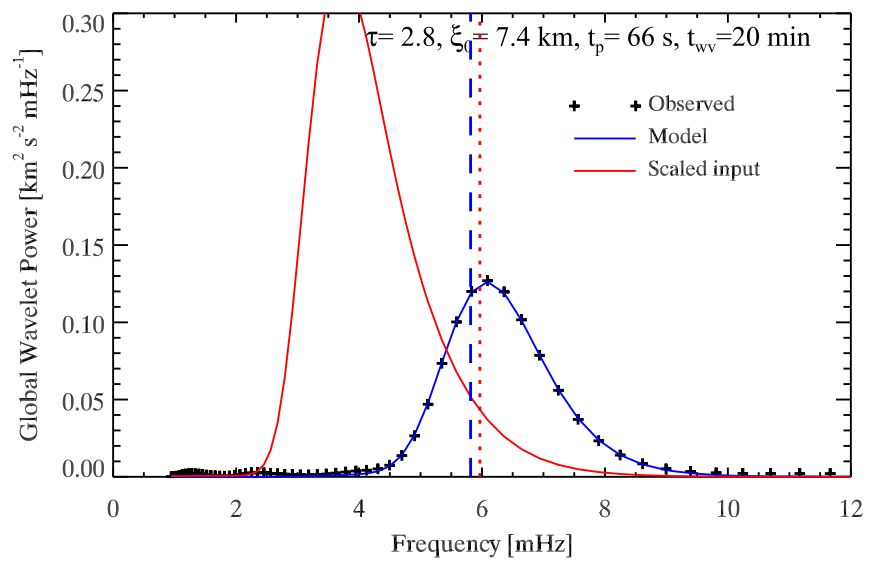

Figure 10. Plots of global wavelet power spectra. The vertical blue dashed line refers to the Lamb frequency $\omega_{1} / 2 \pi$, which corresponds to $c_{1}$, and the vertical red dotted refers to the acoustic cutoff frequency $\omega_{\mathrm{ac}} / 2 \pi$.

Table 1

Parameters of the Theoretical Model Used to Interpret the Observations



\subsection{Model Fitting}

At a given height $z_{\mathrm{D}}$ in the two-layer atmosphere described in Section 4.1, one can calculate velocity $v$ as a function of time $t$ using the equations given in Section 3. The free parameters of the model to be specified are $\tau, \xi_{0}, t_{p}, t_{0}$, and $t_{w v}$. The calculated velocity time series can be compared with the observation of the $\mathrm{Na}$ I line presented in Figure 1. We have determined the optimal values of $\tau, \xi_{0}$, and $t_{p}$ by fitting the observed global wavelet power spectrum with the model, and that of $t_{0}$ by 


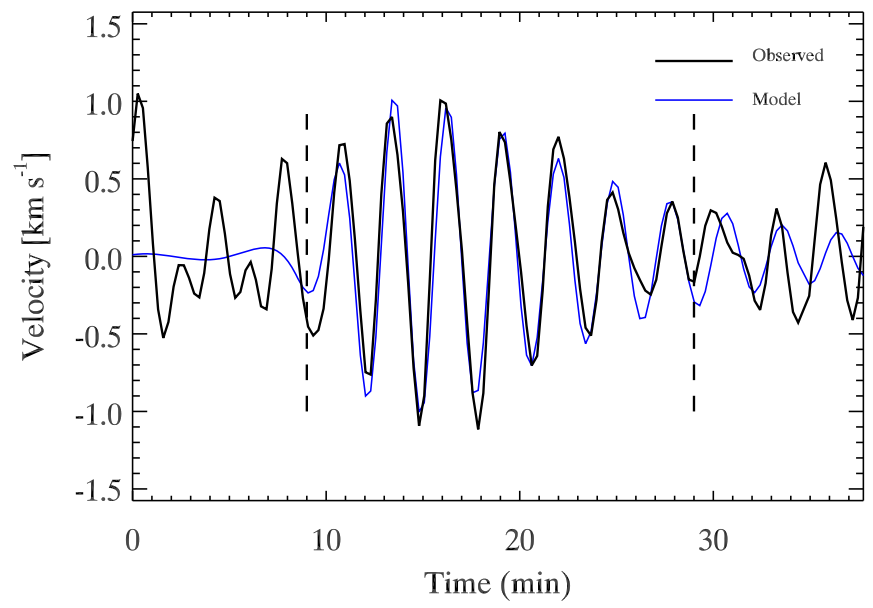

Figure 11. Comparison of the theoretical velocity variation with the observed one. The two vertical dashed lines indicate the start and end of the wave duration.

comparing the velocity variation between the observation and the model. The global wavelet power spectra are the wavelet power spectra averaged over the time span from $t_{0}$ to $t_{0}+t_{w v}$. We set $t_{w v}$ to 20 minutes, which is used to obtain the observed global wavelet power shown in Figure 1.

Figure 10 shows the result of the model fitting of the observed global wavelet power spectrum. The fitting looks quite satisfactory. The determined parameters are $\tau=2.77$, $\xi_{0}=7.4 \mathrm{~km}$, and $t_{p}=66 \mathrm{~s}$. The value of $\tau$ is used to determine the parameters at the lower boundary, namely $c_{0}, z_{0}, h_{0}$, and $p_{0}$. The values of the parameters are listed in Table 1.

The figure also presents the scaled power spectrum of velocity of the driving motion we used as input. The scaling was made by multiplying the square root of the density ratio between $z_{0}$ and $z_{\mathrm{D}}$. If the medium were to allow only progressive waves with height-invariant group velocity, then the power spectrum of the velocity at $z_{\mathrm{D}}$ would be the same as the scaled input spectrum, which in fact is not the case, as can be seen from the figure. This discrepancy between the two power spectra is a clue to understanding how the medium affects the waves. First, we find that the velocity power at $z_{\mathrm{D}}$ is much stronger than the input power spectrum at frequencies $\sim \omega_{\mathrm{ac}} / 2 \pi$. This results from the resonance in the photosphere. We also find that the velocity power at $z_{\mathrm{D}}$ is significantly suppressed at low frequencies $<\omega_{\mathrm{ac}} / 2 \pi$, which is due to the acoustic cutoff. This implies that the velocity power at $z_{\mathrm{D}}$ is insensitive to the input power spectrum at frequencies $\ll \omega_{\text {ac }} / 2 \pi$. Finally, the two power spectra are similar to each other in that they both decrease with frequency at high frequencies.

Figure 11 illustrates the result of such a model calculation compared with the observed velocity profile with the choice of $t_{0}$ in Table 1 . We find that the model is very similar to the observation in period, phase, and amplitude modulation. This successful match supports the notion that each wave packet that is characterized by the amplitude modulation of 10 to 20 minutes results from a single event of wave excitation (Chae et al. 2017).

Figure 12 presents the plot of the flux spectrum $F_{\nu} \equiv 2 \pi F_{\omega}$ with $F_{\omega}$ calculated using Equation (62). The figure shows that the flux spectrum is sharply peaked at a frequency very close to the frequency $\omega_{1} / 2 \pi$, and has a more enhanced wing in the

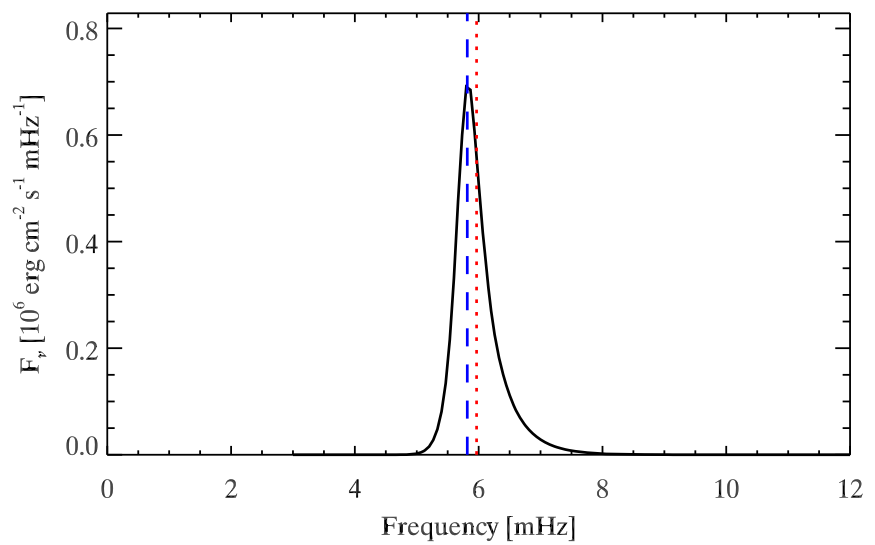

Figure 12. Plots of the flux spectrum. The vertical blue dashed line refers to the Lamb frequency $\omega_{1} / 2 \pi$, which corresponds to $c_{1}$, and the vertical red dotted line refers to the acoustic cutoff frequency $\omega_{\mathrm{ac}} / 2 \pi$.

higher frequency side than in the lower frequency side. The total flux integrated over frequency is found to be $F=$ $0.45 \times 10^{6} \mathrm{erg} \mathrm{cm}^{-2} \mathrm{~s}^{-1}$.

We have also examined the case of $z_{\mathrm{D}}=500 \mathrm{~km}$ instead of $680 \mathrm{~km}$. We found that the fitting of the observed global wavelet power spectrum is as good as in the above case, with the new values $\tau=2.82, \quad \xi_{0}=8.7 \mathrm{~km}, t_{p}=56 \mathrm{~s}$, and $t_{0}=9.1 \mathrm{~min}$, which are slightly different from the above values. The most noticeable change occurs in the values $p_{\mathrm{D}}=10^{3.06}$ and $F=2.6 \times 10^{6} \mathrm{erg} \mathrm{cm}^{-2} \mathrm{~s}^{-1}$. Thus we conclude that the uncertainty of $z_{\mathrm{D}}$ does not affect the general validity of our model, but is critical in the determination of $F$.

\section{Discussion}

\subsection{The Properties of the Model}

In this paper, we have presented the photospheric resonator model of linear acoustic waves in a nonthermal atmosphere, with the goal of modeling three-minute oscillations in the chromosphere of sunspot umbrae. Specifically, we developed a new solution that describes the propagation of acoustic waves in a model atmosphere composed of two layers: one temperature-decreasing layer, and one temperature-increasing layer. Although a realistic description may demand a more detailed multilayer modeling, this simplest two-layer model already reveals a number of observationally significant features.

Our results indicate that when a wave driver is located just below the surface, the photosphere acts as a leaky resonator, bounded by the driver at the bottom and by the temperature minimum region at the top. The temperature minimum region becomes the interface between the two layers, which partially transmits and partially reflects the waves incident from below. The three-minute oscillations observed in the $\mathrm{Na}_{2}$ line can be successfully explained as the progressive waves emitted by the photospheric resonator that is driven by a subphotospheric pulse of vertical displacement.

The most important outcome of our study is how the peak frequency of the velocity power spectrum $\nu_{p}$ is to be interpreted. We have shown that it is close to the Lamb frequency $\omega_{1} / 2 \pi$ of the temperature minimum region. In the specific observation we analyzed, we obtained

$$
\nu_{p}=k \frac{\omega_{1}}{2 \pi}
$$


with a factor $k$ that is close to unity. In our experiment, $k=1.05$ is a good choice. Assuming that this relation holds generally, we can determine from the observed value of $\nu_{p}$ the temperature of the temperature minimum region $T_{1}$ using the formula

$$
\begin{aligned}
T_{1} & =3680 \mathrm{~K}\left[\frac{\gamma}{1.67}\right]\left[\frac{\mu}{1.25}\right]\left[\frac{g}{27,400 \mathrm{~cm} \mathrm{~s}^{-2}}\right]^{2} \\
& \times\left[\frac{k}{1.05}\right]^{2}\left[\frac{\nu_{p}}{6.0 \mathrm{mHz}}\right]^{-2} .
\end{aligned}
$$

For instance, we have obtained $\nu_{p}=6.1 \mathrm{mHz}$ in an umbral region. The observation of Chae et al. (2018) produced an average peak period of 2.89 minutes or $\nu_{p}=5.8 \mathrm{mHz}$ from more Na I line data. By adopting $\gamma=1.67, \mu=1.25$, and $g=27,400 \mathrm{~cm} \mathrm{~s}^{-2}$, we obtain $T_{1}=3560$ and $3940 \mathrm{~K}$. Thus our study provides an independent constraint on the models of the sunspot atmosphere (e.g., Maltby et al. 1986).

We have investigated the photospheric resonator model in the low atmosphere composed of the photosphere and the low chromosphere by adopting the assumption that only progressive waves exist in the chromospheric layer. There are two reasons for this approach. First, our linear theory cannot be used to investigate the behavior of waves at high altitudes. In this upper atmosphere the waves may become highly nonlinear and develop into shock waves, so the linear theory is not applicable (e.g., Chae et al. 2017; Litvinenko \& Chae 2017). Second, we wished to exclude the chromospheric resonator resulting from the reflection from the chromosphere-corona transition layer to focus on the photospheric resonator. The success of the simple two-layer model in explaining the $\mathrm{Na} \mathrm{D}_{2}$ line observations supports the validity of this assumption and suggests that the regressive waves originating from the reflection at the chromosphere-corona transition region may have much less power than the progressive waves. This is in line with the observations (e.g., Cho et al. 2015).

Our model is directly applicable to sunspot umbrae where the magnetic field is very strong and vertical. Slow magnetoacoustic waves in such regions are practically acoustic waves that propagate along the field lines. The driving at the bottom of the atmosphere may be physically identified with the excitation of slow waves by the fast magnetoacoustic waves incident from below, which is often called the process of mode conversion (Cally 2001). Our model may be also applicable to the internetwork regions of the quiet Sun, a practically fieldfree medium, where acoustic waves are observationally manifest as the three-minute oscillations if the driving process occurs near the photosphere. Our model, however, may not be directly applicable to network regions and plage regions where plasma $\beta$ may not be small in the low atmosphere. In these regions, a generalization of the model may be required to include the dependence on magnetic field strength.

\subsection{Comparison with Other Models}

The photospheric resonator model is not the only model that can explain the peak of the power spectrum around the acoustic cutoff frequency determined by the minimum temperature. The chromospheric resonator model as well as the propagating wave model also reproduce the peak.

The photospheric resonator model differs from the chromospheric resonator model in the number of peaks. The chromospheric resonator model predicts a few more peaks above the cutoff, closely spaced in frequency $(1 \mathrm{mHz})$ in addition to the peak near the cutoff. We note that unless spatiotemporally averaged, the observed power spectrum of the chromospheric velocity oscillation is characterized by a single dominant peak around the acoustic cutoff of the temperature minimum, in agreement with our photospheric resonator model. The wavelet power spectrum given in Figure 1 is an example of spatially and temporally resolved power spectra. This power spectrum is characterized by a single peak. More wavelet power spectra of velocity determined from other spectral lines taken either from ground or from space are given in Figure 4 of Chae et al. (2017) and Figures 5 and 7 of Chae et al. (2018). These spectra indicate a successive occurrence of oscillation packets, each of which typically lasts 15 minutes. During the middle of each oscillation packet, the wavelet power spectrum usually has only one peak, which does not agree with the chromospheric resonator model. An exception occurs when the nonlinearity highly develops to be manifest as steepened oscillation profiles. In this case, the second harmonic and higher-order harmonics occur simultaneously with the fundamental peak.

The peak frequency is found to change slightly from packet to packet, so that the time-averaged wavelet power spectra (which are often called global wavelet power spectra and are equivalent to the Fourier power spectra) should be characterized by multiple peaks that are closely spaced in frequency. Because the temperature structure may differ from region to region, the power spectra that are further averaged over space may be found to have a larger number of peaks. This characteristic of the dependence on the spatiotemporal average was clearly illustrated by the observations of Christopoulou et al. (2003) as well.

The photospheric resonator model may explain the frequency variation from oscillation packet to oscillation packet. As clearly illustrated in Figure 11, the photospheric resonator model can successfully reproduce each oscillation packet. The frequency variation can be attributed either to the temporal change of temperature structure with a timescale of several tens of minutes, or to the transverse motion of fine structures of different temperature across the observing point. The first possibility has not been investigated yet, as far as we know, and the other possibility is quite likely because it is observationally well known that an umbra includes a number of fine inhomogeneous structures (such as umbral dots and umbral cores) that often move at significant speeds (e.g., Solanki 2003). Chae et al. (2018), for instance, reported a standard deviation of 0.3 minutes $(0.6 \mathrm{mHz})$ and a mean of 2.89 minutes $(5.8 \mathrm{mHz})$ in the peak period of the oscillation packet observed through Na I D2. With Equation (68), this variation of the peak frequency can be interpreted as the variation of the minimum temperature, with the mean being $3940 \mathrm{~K}$ and the standard deviation being $820 \mathrm{~K}$. A temperature variation of this amount can be easily explained by the variation of the fine features.

The photospheric resonator model and the propagating wave model have subtle differences. In the chromosphere above the temperature minimum, there is no fundamental difference between the two models. In both, waves propagate without much reflection in the chromosphere, and their oscillation spectrum has a single peak. The two models differ from each other mainly in the photosphere, where the photospheric resonator is located. In the propagating wave model that does 
not take reflection at the temperature minimum into account, the wave power should be either constant or decrease with height. In contrast, in the photospheric resonator model, the wave power at frequencies around the cutoff is expected to increase with height because of the reflection at the temperature minimum (see Figure 8). In other words, the wave power can be accumulated and amplified in the photospheric layer. We can observationally distinguish between the propagating wave model and the photospheric resonator model when the waver power at frequencies around the cutoff is determined as a function of height.

We greatly appreciate the referee's constructive comments. This work was supported by the National Research Foundation of Korea (NRF-2017R1A2B4004466), and by the Korea Astronomy and Space Science Institute under the R\&D program, Development of a Solar Coronagraph on International Space Station (Project No. 2017-1-851-00), supervised by the Ministry of Science and ICT.

\section{ORCID iDs}

Jongchul Chae (i) https://orcid.org/0000-0002-7073-868X

Juhyung Kang (i) https://orcid.org/0000-0003-3540-4112

Yuri E. Litvinenko (i) https://orcid.org/0000-0002-1845-5877

\section{References}

Beckers, J. M., \& Tallant, P. E. 1969, SoPh, 7, 351

Botha, G. J. J., Arber, T. D., Nakariakov, V. M., \& Zhugzhda, Y. D. 2011, ApJ, 728, 84

Brynildsen, N., Maltby, P., Brekke, P., et al. 2003, AdSpR, 32, 1097

Brynildsen, N., Maltby, P., Foley, C. R., Fredvik, T., \& Kjeldseth-Moe, O. 2004, SoPh, 221, 237

Cally, P. S. 2001, ApJ, 548, 473

Centeno, R., Collados, M., \& Trujillo Bueno, J. 2006, ApJ, 640, 1153

Chae, J., Cho, K., Song, D., et al. 2018, ApJ, 854, 127

Chae, J., \& Goode, P. R. 2015, ApJ, 808, 118

Chae, J., Lee, J., Cho, K., et al. 2017, ApJ, 836, 18
Chae, J., \& Litvinenko, Y. 2018, ApJ, 869, 36 (Paper I)

Cho, K.-H., Chae, J., Lim, E., \& Yang, H. 2019, ApJ, 879, 67

Cho, K.-S., Bong, S.-C., Nakariakov, V. M., et al. 2015, ApJ, 802, 45

Christopoulou, E. B., Skodras, A., Georgakilas, A. A., et al. 2003, ApJ, 591,416

Felipe, T. 2019, A\&A, 627, A169

Felipe, T., Khomenko, E., Collados, M., \& Beck, C. 2010, ApJ, 722, 131

Fleck, B., \& Schmitz, F. 1991, A\&A, 250, 235

Giovanelli, R. G. 1972, SoPh, 27, 71

Gurman, J. B. 1987, SoPh, 108, 61

Gurman, J. B., \& Leibacher, J. W. 1984, ApJ, 283, 859

Kalkofen, W., Rossi, P., Bodo, G., \& Massaglia, S. 1994, A\&A, 284, 976

Kang, J., Chae, J., Nakariakov, V. M., et al. 2019, ApJL, 877, L9

Khomenko, E., \& Collados, M. 2015, LRSP, 12, 6

Kneer, F., Mattig, W. V., \& v Uexküll, M. 1982, A\&A, 102, 147

Kwak, H., Chae, J., Song, D., et al. 2016, ApJL, 821, L30

Lamb, H. 1909, Proc. London Math. Soc., 7, 122

Lamb, H. 1932, Hydrodynamics (New York: Dover)

Leibacher, J. W., \& Stein, R. F. 1981, NASSP, 263

Lites, B. W. 1986, ApJ, 301, 1005

Lites, B. W., \& Thomas, J. H. 1985, ApJ, 294, 682

Lites, B. W., White, O. R., \& Packman, D. 1982, ApJ, 253, 386

Litvinenko, Y., \& Chae, J. 2017, A\&A, 599, A15

Maltby, P., Avrett, E. H., Carlsson, M., et al. 1986, ApJ, 306, 284

Scheuer, M. A., \& Thomas, J. H. 1981, SoPh, 71, 21

Settele, A., Staude, J., \& Zhugzhda, Y. D. 2001, SoPh, 202, 281

Solanki, S. K. 2003, A\&ARv, 11, 153

Song, D., Chae, J., Kwak, H., et al. 2017, ApJL, 850, L33

Stangalini, M., Giannattasio, F., Del Moro, D., \& Berrilli, F. 2012, A\&A, 539, L4

Staude, J., Zhugzhda, I. D., \& Locans, V. 1985, SoPh, 95, 37

Sutmann, G., \& Ulmschneider, P. 1995, A\&A, 294, 232

Taroyan, Y., \& Erdélyi, R. 2008, SoPh, 251, 523

Thomas, J. H., \& Scheuer, M. A. 1982, SoPh, 79, 19

Tian, H., DeLuca, E., Reeves, K. K., et al. 2014, ApJ, 786, 137

Tritschler, A., \& Schmidt, W. 2002, A\&A, 388, 1048

Uchida, Y., \& Sakurai, T. 1975, PASJ, 27, 259

Zhugzhda, I. D. 1984, MNRAS, 207, 731

Zhugzhda, Y. D. 2007, AstL, 33, 622

Zhugzhda, Y. D. 2018, AstL, 44, 331

Zhugzhda, Y. D., \& Locans, V. 1981, SvAL, 7, 25

Zhugzhda, Y. D., Staude, J., \& Locans, V. 1983, PDHO, 5, 451

Zhugzhda, Y. D., \& Sych, R. A. 2014, AstL, 40, 576 